\title{
Resolving the Galactic ridge X-ray background
}

\author{
M. Revnivtsev ${ }^{1,2}$, A. Vikhlinin ${ }^{3,2}$, and S. Sazonov ${ }^{1,2}$ \\ 1 Max-Planck-Institute für Astrophysik, Karl-Schwarzschild-Str. 1, 85740 Garching bei München, Germany \\ e-mail: mikej@mpa-garching.mpg. de \\ 2 Space Research Institute, Russian Academy of Sciences, Profsoyuznaya 84/32, 117997 Moscow, Russia \\ 3 Harvard-Smithsonian Center for Astrophysics, 60 Garden Street, Cambridge, MA 02138, USA
}

Received 30 November 2006 / Accepted 24 July 2007

\section{ABSTRACT}

\begin{abstract}
We use Chandra deep observations of the Galactic Center (GC) region to improve the constraints on the unresolved fraction of the Galactic ridge X-ray background in the 4-8 keV band. We emphasize the importance of correcting the measured source counts at low fluxes for bias associated with Poisson noise. We find that at distances of $2^{\prime}-4^{\prime}$ from Sgr $\mathrm{A}^{*}$ at least $\sim 40 \%$ of the total X-ray emission in the energy band 4-8 keV originates from point sources with luminosities $L_{2-10 \mathrm{keV}}>10^{31} \mathrm{erg} \mathrm{s}^{-1}$. From a comparison of the source number-flux function in the GC region with the known luminosity function of faint X-ray sources in the Solar vicinity, we infer that Chandra has already resolved a large fraction of the cumulative contribution of cataclysmic variables to the total X-ray flux from the GC region. This comparison further indicates that most of the yet unresolved X-ray flux from the GC region $(\sim 60 \%)$ is likely produced by weak cataclysmic variables and coronally active stars with $L_{2-10 \mathrm{keV}}<10^{31} \mathrm{erg} \mathrm{s}^{-1}$. We conclude that the bulk of the high energy Galactic X-ray background in this direction is produced by discrete sources, and that this is likely to be the case for the rest of the Galactic ridge.
\end{abstract}

Key words. stars: binaries: general - X-rays: binaries - X-rays: general - X-rays: stars - Galaxy: center

\section{Introduction}

One of the largest extended features of the X-ray sky at energies above $\sim 2 \mathrm{keV}$ is the Galactic ridge $\mathrm{X}$-ray emission (GRXE hereafter), discovered in the late 1970's (Cooke et al. 1970; Bleach et al. 1972; Worrall et al. 1982). The GRXE extends over more than 100 degrees along the Galactic plane but only a few degrees across it (e.g., Warwick et al. 1985; Yamauchi \& Koyama 1993).

The origin of the GRXE is the topic of a long-standing debate. The GRXE spectrum resembles the optically thin emission of thermal plasma with a temperature of 5-10 keV (e.g. Koyama et al. 1986, 1989). However, such hot plasma cannot be gravitationally bound to the Galaxy, as suggested by strong concentration of the GRXE towards the Galactic disk and bulge. A number of models, still assuming a diffuse origin of the GRXE, were proposed - magnetic reconnection (e.g. Makishima 1997; Tanuma et al. 1999), charge exchange reactions with cosmic rays (e.g. Tanaka et al. 1999), and interaction of low-energy cosmic-ray electrons with interstellar matter (e.g. Valinia et al. 2000), but none of them was successful in explaining all of the observed properties of the GRXE (see Tanaka 2002, for a review).

An alternative explanation of the GRXE, proposed soon after its discovery, is that it is the superposition of weak Galactic X-ray sources (e.g. Worrall et al. 1982; Worrall \& Marshall 1983; Koyama et al. 1986; Ottmann \& Schmitt 1992; Mukai \& Shiokawa 1993). However, until recently there remained a large uncertainty with regard to the expected contributions of different classes of faint Galactic X-ray sources to the GRXE.

In recent work of Revnivtsev et al. (2006b), Revnivtsev et al. (2006a), and Krivonos et al. (2007), based on X-ray observations from the RXTE and INTEGRAL satellites, it was demonstrated that the GRXE in the energy band 3-60 keV closely traces the near-infrared emission and consequently the stellar mass distribution in the Galaxy. Furthermore, the observed GRXE to stellar mass ratio is compatible with the X-ray luminosity function of cataclysmic variables and coronally active stars in the vicinity of the Sun, measured with RXTE and ROSAT (Sazonov et al. 2006). These findings therefore suggest that the bulk of the GRXE could indeed be produced by emission from discrete sources (Revnivtsev et al. 2006b).

A straightforward way to test the origin of the GRXE is to check which fraction can be resolved into discrete sources in deep Chandra images. The first such study was by Ebisawa et al. $(2001,2005)$ who used a $\sim 200$ ks Chandra observation of a Galactic plane region. They inferred that less than $15 \%$ of the GRXE in that region could be resolved into point sources with fluxes higher than $(3-5) \times 10^{-15} \mathrm{erg} \mathrm{s}^{-1} \mathrm{~cm}^{-2}$.

A good place for studying the GRXE is the Galactic Center (GC) region, because:

- the high concentration of X-ray sources near the GC implies that most of them are located within the Galactic nuclear stellar cluster and thus at the known distance from the Sun; this allows one to easily convert the source $\log N-\log S$ relation to the intrinsic luminosity function and to compare it with the known luminosity function of X-ray sources in the Solar environment;

- the GRXE spectrum in this region is fairly typical (e.g., Tanaka 2002). The X-ray spectrum of unresolved emission in the GC region demonstrates all properties that have been observed in the Galactic plane away from the center (apart from some additional contribution from supernova remnants to the GC region spectrum). The proportionality of the broadband X-ray flux and the flux of the characteristic iron-line emission complex at $\sim 6.7 \mathrm{keV}$ to the near-infrared light of the Galaxy has been firmly established in the GC region 


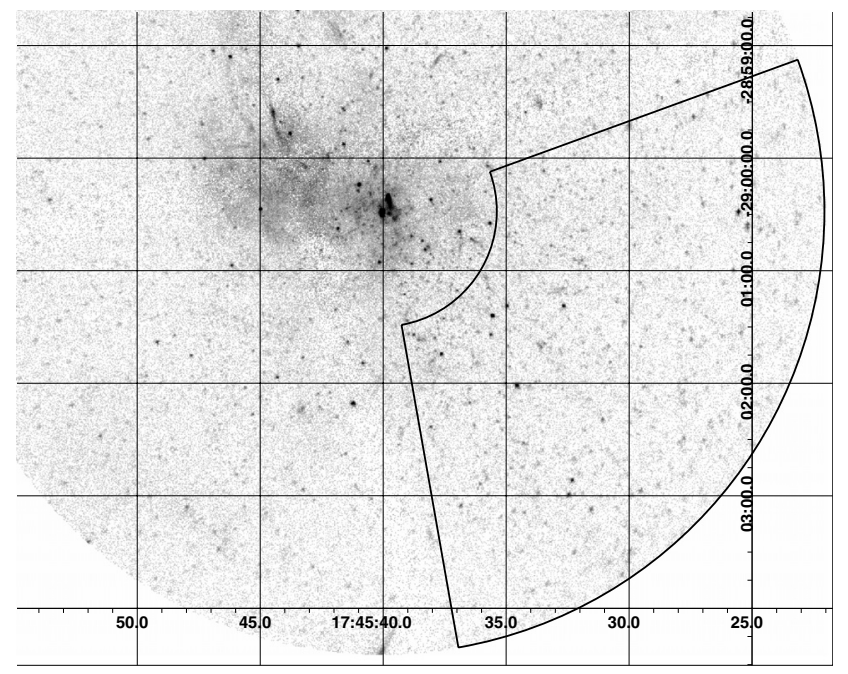

Fig. 1. Chandra image of the GC region in the energy band 4-8 keV (efficiency corrected). The area of our study is outlined by the solid line.

(e.g. Neronov et al. 2005; Warwick et al. 2006), similarly to Galactic plane regions (Revnivtsev et al. 2006b; Revnivtsev et al. 2006a). Thus in practically every respect the "background" emission in this region has the same properties as the "background" emission in Galactic plane regions.

- the GRXE intensity is so high that the contribution of the extragalactic X-ray and the instrumental backgrounds is very low, thus there is virtually no problem in estimating their contribution to the total unresolved emission;

- up to now huge exposure time, approximately $1 \mathrm{Ms}$, was collected by the Chandra observatory in this area.

A disadvantage of the GC region for the GRXE studies is the presence of a large number of supernova remnants and different types of non-thermal phenomena (Muno et al. 2004; Park et al. 2004). However, one can diminish the contribution of these components by considering harder energy ranges.

Chandra has accumulated a long exposure time in the several $\operatorname{arcmin}^{2}$ near the GC. Previous analyses of the combined $\sim 600$ ks worth of data (Muno et al. 2003, 2004; Park et al. 2004) have shown that $\sim 20-30 \%$ of the total X-ray flux can be resolved into point sources in a sub-region that is maximally free from supernova remnants (region "Close" in Muno et al. 2004). Since these works, an additional $330 \mathrm{ksec}$ of GC observations have become publicly available, which allows one to go deeper in resolving the Galactic X-ray background. In this paper, we analyze all available Chandra data in the GC region in an attempt to resolve as much of the GXRE as possible.

\section{Data reduction}

We used Chandra ACIS-I observations of the GC region with the aim point close to Sgr A* (observation ID \#945, 1561, 2282, 2284, 2287, 2291, 2293, 2943, 2951, 2952, 2953, 2954, 3392, 3393, 3663, 3665, 4500, 4683, 4684, 5360, and 6113). The Chandra data were reduced following a standard procedure fully described in Vikhlinin et al. (2005). The only difference is that the detector background was modeled using the stowed dataset (http://cxc.harvard.edu/contrib/maxim/stowed). The total clean exposure time is $918 \mathrm{ks}$. Figure 1 shows the combined image in the $4-8 \mathrm{keV}$ energy band $4-8 \mathrm{keV}$.
The GC region is known to be rich in supernova remnants (see e.g. Park et al. 2004; Muno et al. 2004). In order to minimize the contribution of X-ray emission from plasmas heated by the supernova remnants, in our analysis we 1) considered only a sub-region south-west of the GC (see Fig. 1), subtending $9.77 \mathrm{arcmin}^{2}$, where the contribution of low-temperature plasmas is known to be small from the weakness of low-energy X-ray lines (see e.g. Park et al. 2004), and 2) used only the energy band $4-8 \mathrm{keV}$.

To maximize the sensitivity to point sources, we used only the data within $R<4^{\prime}$ from the optical axis, where the Chandra angular resolution is within $1^{\prime \prime}(F W H M)$. Using a stacked image of bright ( $>100 \mathrm{cnts}$ ) sources, we verified that the effective point spread function (PSF) in the combined image can be well modeled as a Gaussian with $\sigma \sim 0.51^{\prime \prime}$.

\section{Source counting strategy}

To determine the total point source flux we cannot simply coadd the observed counts from detected sources because of strong statistical biases discussed below. A better approach is to integrate the reconstructed $\log N-\log S$ function down to the sensitivity limit.

Because of its excellent angular resolution and low instrumental background, Chandra can detect point sources yielding just a few total counts. Such low detection thresholds lead to strong biases in the derived $\log N-\log S$ function related to the Poisson counting statistics. Generally, the raw number-flux relation near the detection threshold is significantly below the true $\log N-\log S$ because of these effects. This observational bias and methods for correcting it have been extensively studied (e.g. Hasinger et al. 1993; Vikhlinin et al. 1995, and references therein), and in particular for the Chandra image analysis in Moretti et al. (2002); Kenter \& Murray (2003); Bauer et al. (2004). An additional bias in our case arises from source confusion, non-neglibile in the GC region even with the Chandra's angular resolution (Fig. 2).

Our procedure for recovering the true $\log N-\log S$ function in the GC region is based on Monte-Carlo simulations, which allows us to derive accurate corrections for the statistical and source confusion biases. We start with simulating the images containing realistic parent point source populations. We assume that the shape of the $\log N-\log S$ relation is independent of distance from Sgr A* (which corresponds to distance-independent luminosity function in this region). The normalization of the parent $\log N-\log S$ is assumed to vary as $\mathrm{d} N / \mathrm{d} \Omega \propto R^{-1}$, consistent with the previous studies of both the discrete source populations (see e.g. Muno et al. 2003, 2006) and the total X-ray flux (e.g. Neronov et al. 2005) in the GC region. The shape of the parent $\log N-\log S$ is assumed to be a power law, $\mathrm{d} N / \mathrm{d} S \propto S^{-\alpha}$ with $\alpha=3.0$, at bright fluxes $(f>40 \mathrm{cnt})^{1}$. At lower fluxes, we allowed for a nearly arbitrary shape of the intrinsic $\log N-\log S$. Namely, we assumed that $S^{2} \mathrm{~d} N / \mathrm{d} S=$ const. in the flux intervals $40-10,10-3,3-1$, and $1-0.3 \mathrm{cnts}$ but the normalization within each interval is arbitrary. The normalizations of the parent $\log N-\log S$ in each flux range were varied randomly in each realization. For each simulated source, the number of detected photons was drawn from the Poisson distribution.

\footnotetext{
1 Assuming the power law spectrum with the photon index $\Gamma=2$ absorbed with $N_{\mathrm{H}} \sim 5 \times 10^{22} \mathrm{~cm}^{-2}$ (Muno et al. 2004), 40 counts in the 4-8 keV band corresponds to an unabsorved flux of $7 \times$ $10^{-15} \mathrm{erg} \mathrm{s}^{-1} \mathrm{~cm}^{-2}$ in the $2-10 \mathrm{keV}$ band, which corresponds to the luminosity $4.8 \times 10^{31} \mathrm{erg} \mathrm{s}^{-1}$ at the adopted distance to the GC $7.6 \mathrm{kpc}$ (Eisenhauer et al. 2005).
} 


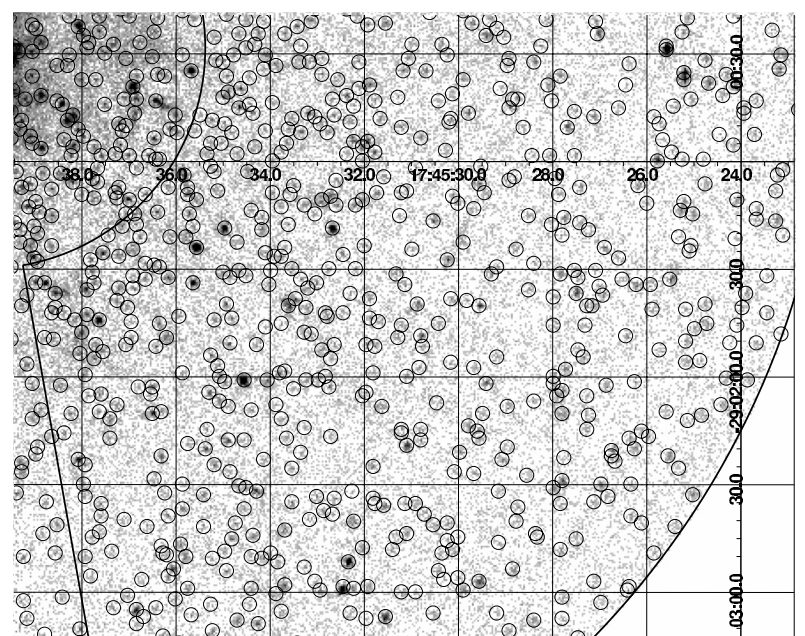

Fig. 2. Part of the image of the GC region in the energy band 4-8 keV, with the detected sources shown by circles (note that the source localizations are actually much more accurate than $\sim 2.5$ arcsec, the circle radius).

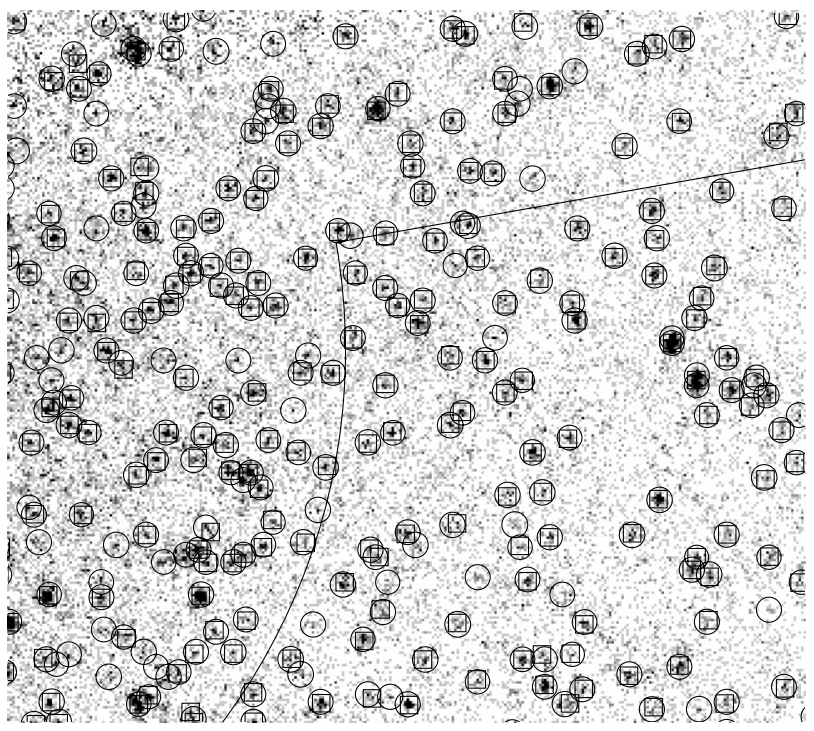

Fig. 3. Comparison of source detections by wavelet decomposition (the task wvdecomp of ZHTOOLS, circles) and the CIAO task wavdetect (boxes). Most of the sources are found by both detection algorithms.

On top of the counts created by simulated sources we added instrumental background at the level of $10^{-7} \mathrm{cnts} / \mathrm{s} / \mathrm{pix}$ in energy band 4-8 keV (e.g. Markevitch et al. 2003), but in the region of our analysis this background practically has no influence on the detection of sources because real photon background (or contribution of unresolved sources, which are weaker than the detection threshold) in the GC region strongly surpasses the instrumental background.

If the total number of simulated counts (including instrumental background counts) was below the total observed number of detected counts, the "missing" flux was added as a diffuse component with the surface brightness $\propto R^{-1}$. An example of the simulated image is shown in Fig. 4.

Our source detection is based on the wavelet decomposition algorithm described in Vikhlinin et al. (1998). The detection threshold (specified as a required statistical significance) was chosen so that less than 1 spurious detection is allowed over the region of interest. Sources detected in the Chandra image of the GC region are shown by circles in Fig. 2. We also checked that wavelet decomposition provides equal or better sensitivity to the point sources compared with the CIAO task wavdetect (Fig. 3). Both wavdetect and wvdecomp are based on the convolution of the image with a wavelet kernel (note, that this is not what we call "decomposition"). However, the implementations of other essential steps in source detection, for example subtraction of bright sources before a subsequent search for weaker sources, are different (see detailed description of the method of wvdecomp in Vikhlinin et al. 1998). A detailed comparison of the advantages and disadvantages of the two tools is beyond the scope of our work. For our purposes, it was sufficient to show that wvdecomp works at least as well as wavdectect.

The fluxes of detected sources were measured within the $2.5^{\prime \prime}$ aperture, which should contain nearly $100 \%$ of the total source flux for the observed PSF width (see Sect. 2). The local background, provided by the largest scale of the wavelet decomposition, $\sim 16^{\prime \prime}$, was subtracted from the source flux.

The raw differential number-flux relation derived from the real observation is shown by crosses in Fig. 5. From our simulations, we can determine the "response" to which shape and normalization of the parent $\log N-\log S$ best describes the observed flux distribution. These results are discussed below.

\section{Results}

The region outlined by the solid line in Fig. 1 contains in total $63.5 \mathrm{kcnts}$ in the energy band $4-8 \mathrm{keV}$. The estimated particle background image of the same region contains $10.6 \mathrm{kc}-$ nts. Thus, the net background-corrected number of counts is 52.9 kcnts. The total flux from all the sources detected in the region is $\sim 14.3 \mathrm{kcnts}$.

\subsection{Resolved fraction}

Figure 5 shows an example of a simulated number-flux function that is consistent with the distribution measured by Chandra. Also shown is the corresponding intrinsic $\log N-\log S$ relation. By sampling over many such well-matching (the reduced $\chi^{2}$ difference from the observed number-flux function is less than 1.5) trial distributions, we determined the allowed range of the cumulative flux of "parent" sources in the GC region (shaded region in Fig. 5).

The outcome of this analysis is presented in Fig. 6. Integrating from a lower limit of three counts on our image, between $35 \%$ and $80 \%$ of the total flux can be attributed to discrete sources. We note that in obtaining this result we limited ourselves to sources with average intensities of more than 3 integrated counts, because the number densities of yet weaker sources are poorly constrained by the available data. Therefore, $\sim 35 \%$ is actually a conservative lower limit on the contribution of point sources to the total X-ray flux. Below we discuss the expected contribution of sources with luminosities below the Chandra detection threshold.

\subsection{Luminosity function of point $X$-ray sources}

We now address the luminosity function of faint $X$-ray sources in the GC region. Since the volume density of stars within several tens of parsecs of $\mathrm{Sgr} \mathrm{A}^{*}$ is several orders of magnitude higher than in other places along the line of sight in that direction, we can safely assume that the absolute majority of stars and X-ray sources in the sky region of our study (located at $\sim 4^{\prime}$ or at a 


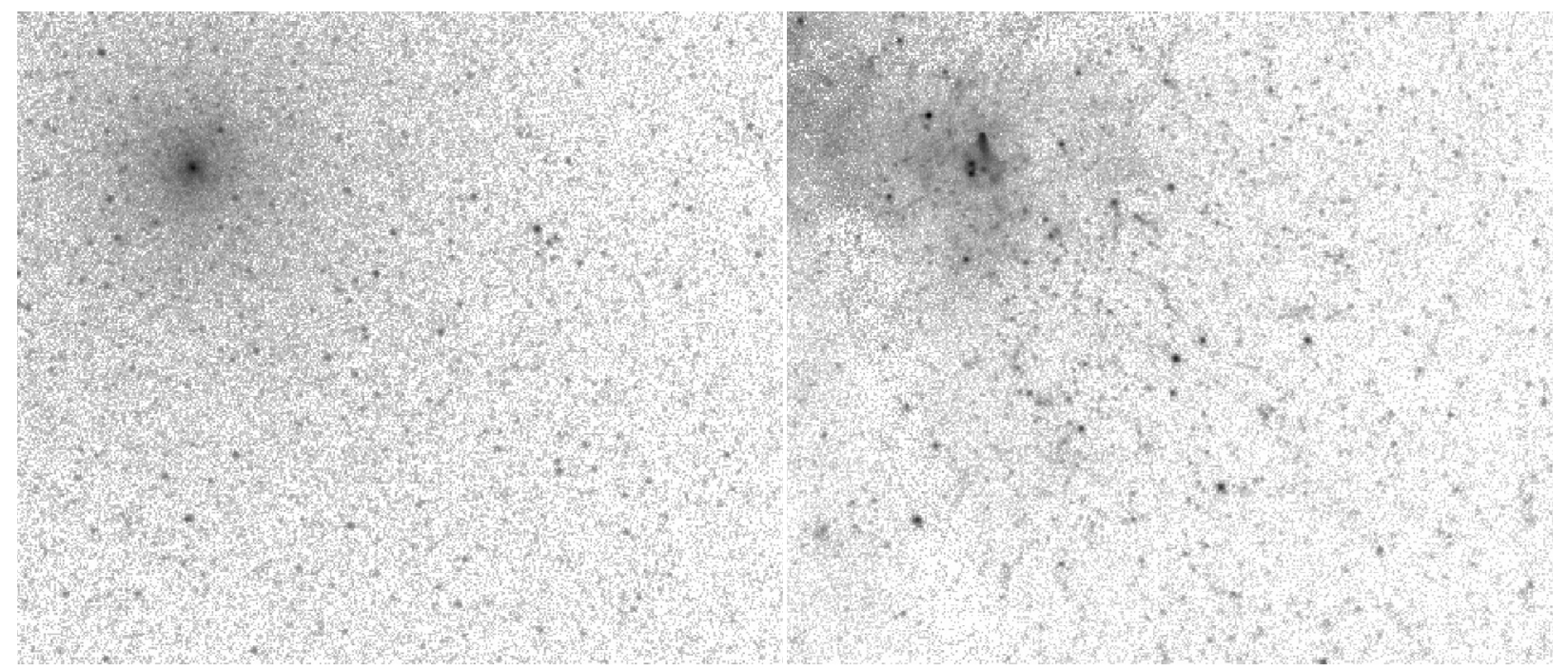

Fig. 4. Simulated image of the GC region (left) vs. the real image taken by Chandra (right). The GC is in the upper left corner.

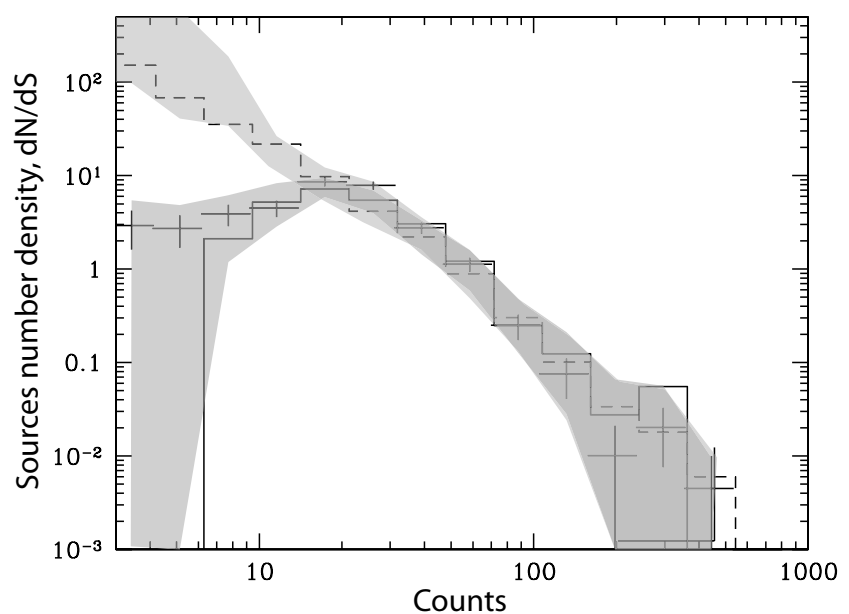

Fig. 5. Differential number-flux function of sources in the region of our study (points with error bars) along with a well-matching simulated number-flux distribution (solid line) and the corresponding parent (before the detection procedure) distribution of sources (dashed line). The lower gray area denotes the manifold of trial shapes of differential luminosity functions of simulated sources, which we considered as satisfactorily describing the observed $\mathrm{d} N / \mathrm{d} S$ function. The upper gray area shows the manifold of possible shapes of the parent $\mathrm{d} N / \mathrm{d} S$ function.

$\sim 10$ pc projected distance from Sgr A*, see Fig. 1) physically belong to either the nuclear stellar cluster (NSC) or nuclear stellar disk (NSD) components of the Galaxy (Genzel \& Townes 1987; Launhardt et al. 2002). This allows us to readily estimate the stellar mass contained in the volume of the Galaxy covered by our observations and consequently the luminosity function of $\mathrm{X}$-ray sources in the $\mathrm{GC}$ region normalized by stellar mass.

For the NSC we adopted the following density profile:

$\rho_{\mathrm{NSC}}=\frac{\rho_{\mathrm{c}}}{1+\left(r / r_{\mathrm{c}}\right)^{n}}$,

where $\rho_{\mathrm{c}}=3.3 \times 10^{6} M_{\odot} / \mathrm{pc}^{3}$ and $r_{\mathrm{c}}=0.22 \mathrm{pc}$. The slope was assumed to be $n=2$ at $r<6$ pc and $n=3$ at larger distances. The total mass of the NSC within 200 pc of Sgr A* is thus $6 \times$ $10^{7} M_{\odot}$. This value is actually uncertain by a factor of $\sim 2$ (see e.g. Lindqvist et al. 1992; Launhardt et al. 2002).

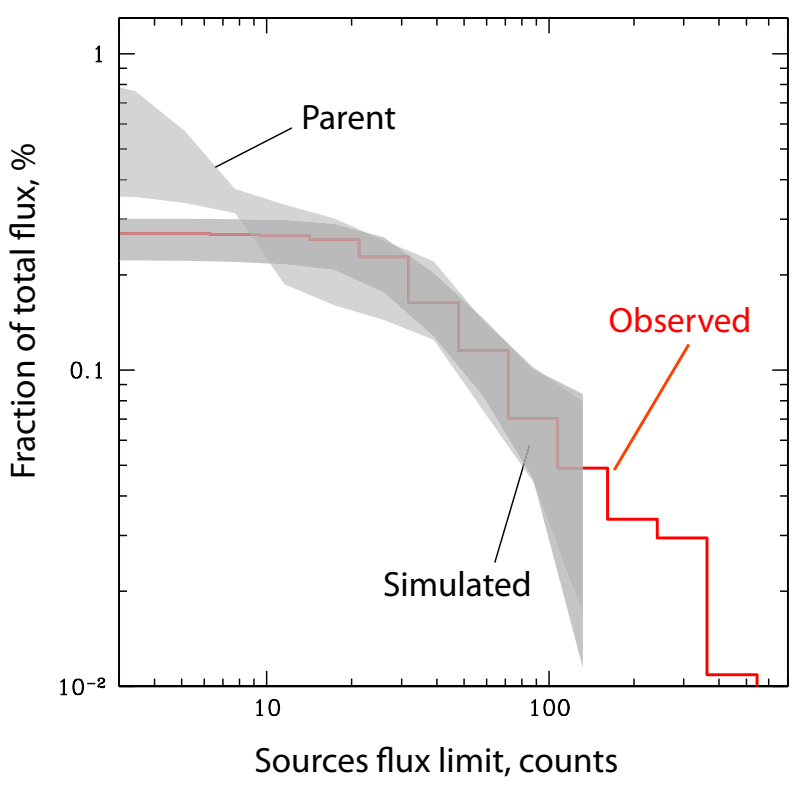

Fig. 6. Cumulative flux (relative to the total flux) from the detected sources in the real and simulated observations and the allowed range for the parent source distribution. Note that one count during the whole observation corresponds to an intrinsic (corrected for the line-of-sight extinction of $N_{\mathrm{H}}=5 \times 10^{22} \mathrm{~cm}^{-2}$ ) source luminosity $\sim 1.3 \times 10^{30} \mathrm{erg} \mathrm{s}^{-1}$.

The NSD was assumed to have the density distribution ( $r$ and $z$ are measured in parsecs)

$\rho_{\mathrm{NSD}}=\rho_{\mathrm{d}} r^{-\alpha} \mathrm{e}^{-|z| / z_{\mathrm{d}}}$,

where $\rho_{\mathrm{d}}=300 M_{\odot} / \mathrm{pc}^{3}$ and $z_{\mathrm{d}}=45 \mathrm{pc}$. At $r<120 \mathrm{pc}$, the slope $\alpha=0.1$, at $120 \mathrm{pc}<r<220 \mathrm{pc}, \alpha=3.5$, and at $r>220 \mathrm{pc}$, $\alpha=10$ (Launhardt et al. 2002). The total mass of the NSD is thus $1.4 \times 10^{9} M_{\odot}$. In reality this quantity is uncertain by some $50 \%$ (Launhardt et al. 2002). We took the distance to the GC to be $7.6 \mathrm{kpc}$ (Eisenhauer et al. 2005).

The adopted mass model gives a total mass of stars enclosed in the region of our study of $\sim 4.4 \times 10^{6} M_{\odot}$. This value is uncertain by a factor of $\sim 2$ mainly due to the uncertainty in the NSC 


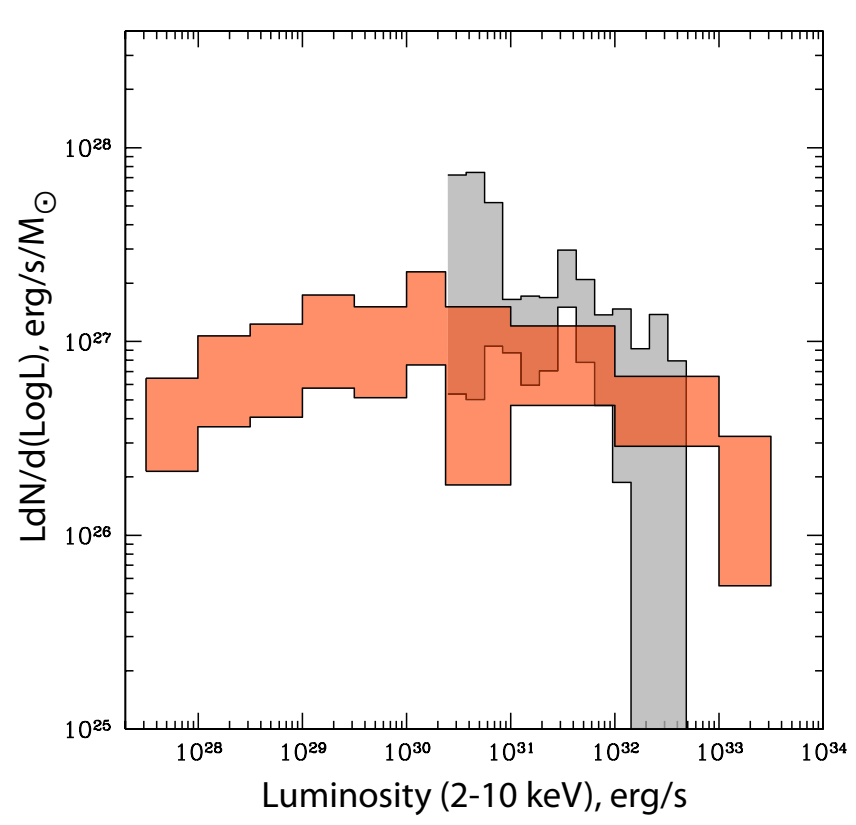

Fig. 7. Luminosity function of weak X-ray sources in the Solar neighborhood (red area) in comparison with the allowed range for the luminosity function in the GC region (grey area). The normalization of the GC luminosity function per unit stellar mass is uncertain by a factor of $\sim 2$ due to the uncertainty in the mass of the NSC.

mass. We note that a similar mass model was found to provide a satisfactory description of the surface density distribution of detected X-ray sources in the GC region (Muno et al. 2006).

Using this estimate of the enclosed stellar mass and the Chandra source number-flux function, we obtained from simulations the allowed range for the luminosity function of X-ray sources in the GC region at luminosities $L_{2-10 \mathrm{keV}}>10^{30} \mathrm{erg} \mathrm{s}^{-1}$, which is shown in Fig. 7. It is important to note that the overall normalization of this luminosity function is uncertain by a factor of $\sim 2$ due to the uncertainty in the stellar mass enclosed in the studied region of the Galaxy. For comparison we show in Fig. 7 (points with error bars) the result of simply dividing the differential $\log N-\log S$ function measured by Chandra by the enclosed stellar mass. This naive determination is affected by the bias discussed in the previous section and thus underestimates the true luminosity function. On the other hand, the allowed range for the luminosity function inferred from the Chandra data for the dense GC region is compatible with the luminosity function of faint X-ray sources in the Solar vicinity (Sazonov et al. 2006, Fig. 7), taking into account the uncertainty in the NSC mass.

The total absorption-corrected (assuming a line-of-sight absorption of $N_{\mathrm{H}}=5 \times 10^{22} \mathrm{~cm}^{-2}$ ) X-ray flux in the energy band $2-10 \mathrm{keV}$ from the studied GC region is $F_{\mathrm{x}}=(5.8 \pm$ $0.4) \times 10^{-12} \mathrm{erg} \mathrm{s}^{-1} \mathrm{~cm}^{-2}$. For the GC distance of $7.6 \mathrm{kpc}$ (Eisenhauer et al. 2005), this corresponds to a total X-ray luminosity $L_{2-10 \mathrm{keV}}=(4.0 \pm 0.2) \times 10^{34} \mathrm{erg} \mathrm{s}^{-1}$. Therefore the total X-ray emissivity in the studied volume of the Galaxy is $L_{\mathrm{x}} / M=(9.1 \pm 4.6) \times 10^{27} \mathrm{erg} \mathrm{s}^{-1} M_{\odot}^{-1}$, where we included the uncertainty in the NSC mass. This derived value of the $\mathrm{X}$-ray emissivity per unit stellar mass agrees within the uncertainties with the cumulative emissivity of faint X-ray sources (cataclysmic variables and coronally active stars) near the Sun: $(4.5 \pm 0.9) \times 10^{27} \mathrm{erg} \mathrm{s}^{-1} M_{\odot}^{-1}$ (Sazonov et al. 2006).

We should note here that in the studied GC region there may be a non-negligible contribution from warm diffuse plasma heated by supernova remnants. According to Muno et al. (2004), $\sim 15 \%$ of the total $2-10 \mathrm{keV}$ flux is probably due to a $\sim 0.8 \mathrm{keV}$ plasma. This would mean that the X-ray emissivity of point sources in the GC region is actually somewhat smaller, $L_{\mathrm{x}} / M=$ $(7.7 \pm 3.9) \times 10^{27} \mathrm{erg} \mathrm{s}^{-1} M_{\odot}^{-1}$, i.e., even closer to the value measured by Sazonov et al. (2006) in the Solar neighborhood.

\section{Discussion}

We showed that at least $40 \%$ of the total X-ray emission from the GC region is produced by point sources with luminosities $L_{2-10 \mathrm{keV}}>10^{31} \mathrm{erg} \mathrm{s}^{-1}$. The inferred luminosity function of such sources (see also Muno et al. 2006) is compatible with that measured in the Solar vicinity (Sazonov et al. 2006). Moreover, the data are consistent with the hypothesis that sources with luminosities below $\sim 10^{31} \mathrm{erg} \mathrm{s}^{-1}$, the effective Chandra detection limit, provide the rest of the total X-ray flux. Such sources are in fact expected to be present in the required numbers in the GC region. However, one should keep in mind that the GC region is characterized by an increased number density of supernova remnants with respect to ordinary regions in the Galactic disk, so a slightly different contribution of truly diffuse emission to the total X-ray emission can be expected.

According to studies in the Solar neighborhood (Sazonov et al. 2006), most of the X-ray sources with luminosities $L_{2-10 \mathrm{keV}}<10^{31} \mathrm{erg} \mathrm{s}^{-1}$ are coronally active stars. Late-type stars with convective envelopes can have coronae where the plasma can be heated up to X-ray temperatures (see Güdel 2004, for a review). Such stars are usually fast rotators, as is required for high coronal activity, because they are either members of binary systems or relatively young (see e.g. Walter \& Bowyer 1981; Queloz et al. 1998). The relative fraction of binary stars with active coronae near the Sun is quite high: at least every 80th star has an X-ray $(2-10 \mathrm{keV})$ luminosity in the range $10^{27} \mathrm{erg} \mathrm{s}^{-1}<L_{2-10} \mathrm{keV}<2 \times 10^{30} \mathrm{erg} \mathrm{s}^{-1}$. The relative fraction of single stars with active coronae is even larger.

The relative contribution of coronally active stars to the total $\mathrm{X}$-ray emission of a given volume of the Galaxy is estimated to be $30-60 \%$ (Sazonov et al. 2006). For this fraction to be smaller, the fraction of stars with convective envelopes (usually late-type, low-mass stars) should somehow be reduced, whereas the population of low-mass stars at distances $4.5-8.7$ pc from Sgr A* appears quite normal (e.g. Philipp et al. 1999).

As mentioned above, the luminosity function of GC sources agrees within the uncertainties with that measured in the Solar vicinity (Sazonov et al. 2006). We point out that because the vast majority of sources detected in the GC region are located at the same and known distance from us, a luminosity function determined there can be more accurate than a luminosity function determined elsewhere.

In Fig. 8 we present the photon spectrum in the region of our study along with the spectrum of all detected point sources. In this paper we have not considered the spectra of resolved sources and unresolved emission in detail. The main reason for this is the extreme narrowness of the considered energy band (4-8 keV) and still important (and spatially variable) photoabsorption. Combination of these two flaws prevents virtually any solid conclusions from the study of spectra of resolved sources. We plan to extend our analysis and perform such a study of the GRXE in regions with much smaller (or negligible) photoabsorption (see e.g. regions in van den Berg et al. 2006) in future works. 


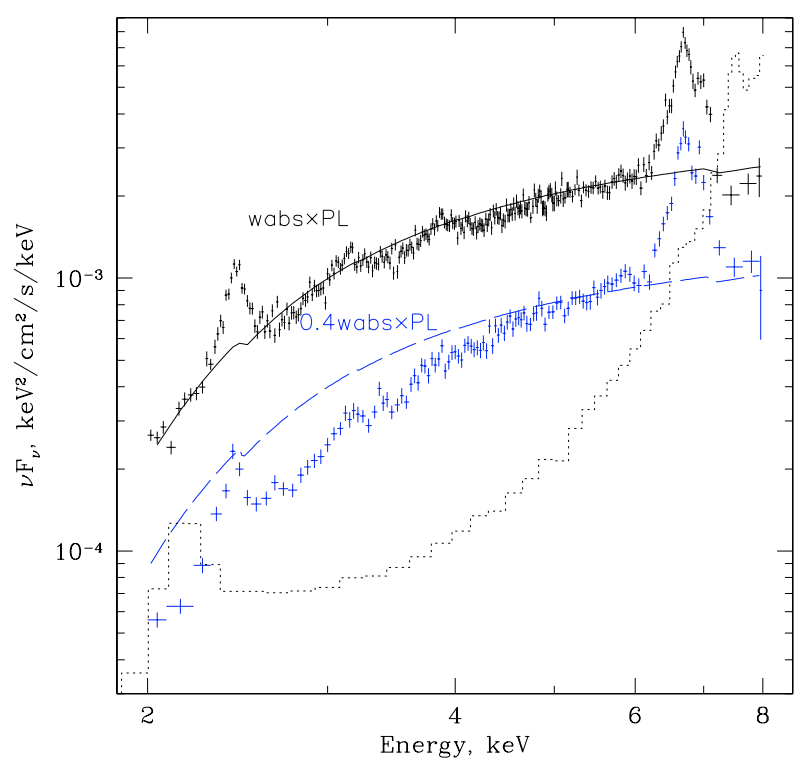

Fig. 8. Spectrum of the studied region (black crosses) along with the spectrum of the estimated CHANDRA/ACIS-I background (dotted histogramm) and cumulative spectrum of all point sources detected in the region (blue crosses). Solid line denotes a power law model $\left(\mathrm{d} N / \mathrm{d} E \propto E^{-1.7}\right)$ with neutral photoabsorption with absorption column $N_{\mathrm{H}}=5 \times 10^{22} \mathrm{~cm}^{-2}$. Dashed line is the same model, multiplied by factor 0.4 .

Acknowledgements. The results reported in this paper have become possible thanks to the the Chandra X-ray Observatory with its superior angular resolution. This research made use of data obtained from the High Energy Astrophysics Science Archive Research Center Online Service, provided by the NASA/Goddard Space Flight Center. M.R. aknowledge partial support of grant RFFI 07-02-00961.

\section{References}

Bauer, F. E., Alexander, D. M., Brandt, W. N., et al. 2004, AJ, 128, 2048 Bleach, R. D., Boldt, E. A., Holt, S. S., Schwartz, D. A., \& Serlemitsos, P. J. 1972, ApJ, 174, L101

Cooke, B. A., Griffiths, R. E., \& Pounds, K. A. 1970, IAUS, 37, 280

Ebisawa, K., Maeda, Y., Kaneda, H., \& Yamauchi, S. 2001, Science, 293, 1633

Ebisawa, K., Tsujimoto, M., Paizis, A., et al. 2005, ApJ, 635, 214
Eisenhauer, F., Genzel, R., Alexander, T., et al. 2005, ApJ, 628, 246

Genzel, R., \& Townes, C. H. 1987, ARA\&A, 25, 377

Güdel, M. 2004, A\&ARv, 12, 71

Hasinger, G., Burg, R., Giacconi, R., et al. 1993, A\&A, 275, 1

Kenter, A. T., \& Murray, S. S. 2003, ApJ, 584, 1016

Koyama, K., Makishima, K., Tanaka, Y., \& Tsunemi, H. 1986, PASJ, 38, 121

Koyama, K., Awaki, H., Kunieda, H., Takano, S., \& Tawara, Y. 1989, Nature, 339, 603

Krivonos, R., Revnivtsev, M., Churazov, E., et al. 2007, A\&A, 463, 957

Launhardt, R., Zylka, R., \& Mezger, P. G. 2002, A\&A, 384, 112

Lindqvist, M., Habing, H. J., \& Winnberg, A. 1992, A\&A, 259, 118

Makishima, K. 1997, X-Ray Imaging and Spectroscopy of Cosmic Hot Plasmas, 137

Markevitch, M., Bautz, M. W., Biller, B., et al. 2003, ApJ, 583, 70

Moretti, A., Lazzati, D., Campana, S., \& Tagliaferri, G. 2002, ApJ, 570, 502

Mukai, K., \& Shiokawa, K. 1993, ApJ, 418, 863

Muno, M. P., Baganoff, F. K., Bautz, M. W., et al. 2003, ApJ, 589, 225

Muno, M. P., Baganoff, F. K., Bautz, M. W., et al. 2004, ApJ, 613, 326

Muno, M. P., Bauer, F. E., Bandyopadhyay, R. M., \& Wang, Q. D. 2006, ApJS, 165,173

Neronov, A., Chernyakova, M., Courvoisier, T. J., \& Walter, R. 2005, [arXiv:astro-ph/0506437]

Ottmann, R., \& Schmitt, J. H. M. M. 1992, A\&A, 256, 421

Park, S., Muno, M. P., Baganoff, F. K., et al. 2004, ApJ, 603, 548

Philipp, S., Zylka, R., Mezger, P. G., et al. 1999, A\&A, 348, 768

Queloz, D., Allain, S., Mermilliod, J.-C., Bouvier, J., \& Mayor, M. 1998, A\&A, 335,183

Revnivtsev, M., Molkov, S., \& Sazonov, S. 2006a, MNRAS, 373, L11

Revnivtsev, M., Sazonov, S., Gilfanov, M., Churazov, E., \& Sunyaev, R. 2006b, A\&A, 452, 169

Sazonov, S., Revnivtsev, M., Gilfanov, M., Churazov, E., \& Sunyaev, R. 2006, A\&A, 450, 117

Schmitt, J. H. M. M., Collura, A., Sciortino, S., et al. 1990, ApJ, 365, 704

Tanaka, Y. 2002, A\&A, 382, 1052

Tanaka, Y., Miyaji, T., \& Hasinger, G. 1999, AN, 320, 181

Tanuma, S., Yokoyama, T., Kudoh, T., et al. 1999, PASJ, 51, 161

Valinia, A., Tatischeff, V., Arnaud, K., Ebisawa, K., \& Ramaty, R. 2000, ApJ, 543,733

van den Berg, M., Grindlay, J., Laycock, S., et al. 2006, ApJ, 647, L135

Vikhlinin, A., Forman, W., Jones, C., \& Murray, S. 1995, ApJ, 451, 542

Vikhlinin, A., McNamara, B. R., Forman, W., et al. 1998, ApJ, 502, 558

Vikhlinin, A., Markevitch, M., Murray, S. S., et al. 2005, ApJ, 628, 655

Worrall, D. M., \& Marshall, F. E. 1983, ApJ, 267, 691

Worrall, D. M., Marshall, F. E., Boldt, E. A., \& Swank, J. H. 1982, ApJ, 255, 111

Walter, F. M., \& Bowyer, S. 1981, ApJ, 245, 671

Warwick, R. S., Turner, M. J. L., Watson, M. G., \& Willingale, R. 1985, Nature, 317,218

Warwick, R., Sakano, M., \& Decourchelle, A. 2006, The 6.7-keV iron-line emission in the Galactic Centre, J. Phys. Conf. Ser. 54, 103

Yamauchi, S., \& Koyama, K. 1993, ApJ, 404, 620 